\title{
Gas Sensors Based on Locally Heated Multiwall Carbon Nanotubes Decorated with Metal Nanoparticles
}

\author{
R. Savu, ${ }^{1}$ J. V. Silveira, ${ }^{1,2}$ A. Alaferdov, ${ }^{1}$ E. Joanni, ${ }^{3}$ A. L. Gobbi, ${ }^{4}$ M. A. Canesqui, \\ D. S. de Lara, ${ }^{1}$ A. G. Souza Filho, ${ }^{2}$ and S. A. Moshkalev ${ }^{1}$ \\ ${ }^{1}$ Centro de Componentes Semicondutores (CCS), Universidade Estadual de Campinas (UNICAMP), CP 6061, \\ Rua João Pandia Calógeras 90, 13083-870 Campinas, SP, Brazil \\ ${ }^{2}$ Departamento de Física, Universidade Federal do Ceará, P.O. Box 6030, 60455-900 Fortaleza, CE, Brazil \\ ${ }^{3}$ Divisão de Tecnologias Tridimensionais (DT3D), Centro de Tecnologia da Informação Renato Archer, Rodovia D. Pedro I Km 143.6, \\ 13069-901 Campinas, SP, Brazil \\ ${ }^{4}$ Laboratório de Microfabricação, Centro Nacional de Pesquisa em Energia e Materiais (CNPEM), \\ Rua Giuseppe Máximo Scolfaro 10.000, Pólo II de Alta Tecnologia, 13083-970 Campinas, SP, Brazil
}

Correspondence should be addressed to R. Savu; raluca.savu@ccs.unicamp.br

Received 28 September 2014; Accepted 6 February 2015

Academic Editor: Xiao-Miao Feng

Copyright (C) 2015 R. Savu et al. This is an open access article distributed under the Creative Commons Attribution License, which permits unrestricted use, distribution, and reproduction in any medium, provided the original work is properly cited.

\begin{abstract}
We report the design and fabrication of microreactors and sensors based on metal nanoparticle-decorated carbon nanotubes. Titanium adhesion layers and gold films were sputtered onto $\mathrm{Si} / \mathrm{SiO}_{2}$ substrates for obtaining the electrical contacts. The gold layers were electrochemically thickened until $1 \mu \mathrm{m}$ and the electrodes were patterned using photolithography and wet chemical etching. Before the dielectrophoretic deposition of the nanotubes, a gap $1 \mu \mathrm{m}$ wide and $5 \mu \mathrm{m}$ deep was milled in the middle of the metallic line by focused ion beam, allowing the fabrication of sensors based on suspended nanotubes bridging the electrodes. Subsequently, the sputtering technique was used for decorating the nanotubes with metallic nanoparticles. In order to test the as-obtained sensors, microreactors $\left(100 \mu \mathrm{L}\right.$ volume) were machined from a single Kovar piece, being equipped with electrical connections and $1 / 4^{\prime \prime}$ Swagelok-compatible gas inlet and outlets for controlling the atmosphere in the testing chamber. The sensors, electrically connected to the contact pins by wire-bonding, were tested in the $10^{-5}$ to $10^{-2} \mathrm{~W}$ working power interval using oxygen as target gas. The small chamber volume allowed the measurement of fast characteristic times (response/recovery), with the sensors showing good sensitivity.
\end{abstract}

\section{Introduction}

Carbon nanotubes (CNTs) are in the top list of most studied nanomaterials due to their exceptional electrical, optical, mechanical, and thermal properties, which make them a model system for nanoscience and nanotechnology [1-4]. Regarding the number of layers, these nanostructures are divided into two main categories: single-walled carbon nanotubes (SWCNTs) and multiwalled carbon nanotubes (MWCNTs) [1]. The decoration of CNTs with nanoparticles (CNTs/NPs), by means of chemical and/or physical methods, to produce novel hybrid nanostructured materials, proved to be a useful approach for engineering their properties and further increasing their range of applications. Therefore, carbon nanostructures, either functionalized or decorated with nanoparticles, are the basis of various novel micro- and nanoscale devices $[1,2,4]$.

Microsensors in a chemiresistor configuration based on carbon nanotubes deposited precisely over metal electrodes from liquid dispersions, using the ac dielectrophoresis process, have been recently reported $[5,6]$. The number of the nanotubes deposited by this method can be controlled by appropriately setting the process conditions, covering a range from several hundreds to an individual nanotube. However, it should be noted that pristine nanotubes usually do not meet the basic gas sensor requirements of high sensibility, selectivity, and short response and recovery times [7]. On the other hand, either functionalized or novel hybrid 
nanostructures, such as carbon nanotubes decorated with metal or metal oxide nanoparticles, proved to be very suitable for gas sensing devices $[5,8-10]$. In this case, the important functions important for gas sensing are separated between the two components of the hybrid material: (1) nanoparticles: interacting strongly with the reactive gas injected in the test chamber, passing electrical charges generated in the reactions directly to the nanotubes; (2) carbon nanotubes serving as supports, local heaters, and low-resistance conductors (transducers). This separation of functions is important to provide a direct response of the sensor to the target gases, since the chemical interaction between gas and particles does not change the electrical conductivity of the nanotubes. Decoration of nanotubes by both metal and semiconductor nanoparticles has been used in our previous works to increase the sensitivity, to decrease the response times, and to improve the selectivity to different gases $[5,6]$.

Microreactors that incorporate the aforementioned sensors have the advantage of combining nanostructured sensing materials with small working volume, thus offering better monitoring and feature controlling of these devices [11]. Moreover, the reactors allow sensor miniaturization and open the possibility of use in many novel applications, such as very compact, noninvasive pressure sensors, accelerometers, and gas sensors $[7,12]$.

In a previous work [13] we reported the design and fabrication of a microreactor that offers the advantages of easy assembly, low cost, and good performance for nanosensor testing, easily fitting any vacuum/gas system and being in effect a mobile, easy to use, multiple test chamber. Using this small testing chamber we performed gas sensor tests based on hybrid nanostructures. The goal of this paper is to report the response of gas sensors based on locally heated multiwall carbon nanotubes decorated with metal nanoparticles.

\section{Experimental Details}

2.1. Deposition of Pristine and Decorated Carbon Nanotubes and Sensor Configuration. The gas sensors were obtained by depositing multiwall carbon nanotubes (CNT Co. Ltd.) over gold patterned electrodes by ac dielectrophoresis (DEP). In order to have a good gold adhesion, a thin metallic Ti film $(30 \mathrm{~nm})$ was first deposited by sputtering over the thermally oxidized silicon substrates. Afterwards, a $150 \mathrm{~nm}$ thick gold layer was sputtered over the adhesion layer and afterwards electrochemically thickened up to $1 \mu \mathrm{m}$. The patterning of the electrodes was made by photolithography followed by wet chemical etching. Prior to deposition, gaps of $1 \mu \mathrm{m}$ wide and $5 \mu \mathrm{m}$ deep were milled in the middle of the metallic lines by focused ion beam (FIB, NOVA 200 Nanolab, FEI Co.). Figure 1(a) shows a schematic drawing of the gas sensors fabricated by depositing CNTs, using the DEP method, onto oxidized silicon substrates with thick gold patterned electrodes. The experimental parameters used for the DEP process were DMF (di-methylformamide) suspensions containing carbon nanotubes, a frequency of $100 \mathrm{kHz}$, a peak-topeak voltage from 0.5 to $3 \mathrm{~V}$, and a deposition time varying from 15 seconds to 3 minutes. On average, the electrical contact between the as deposited nanotubes and the gold electrodes is quite poor. In order to reduce the contact resistance, thermal treatment processes were conducted in a tubular furnace at $300^{\circ} \mathrm{C}$ in inert atmosphere $\left(\mathrm{Ar}\right.$ or $\left.\mathrm{N}_{2}\right)$ for 1 hour.

The hybrid nanostructures were obtained by depositing thin $\mathrm{Ti}$ or $\mathrm{Cu}$ films over the carbon nanotubes using the plasma sputtering technique. The deposition conditions used were room temperature, $2 \times 10^{-2}$ mbar Ar pressure, $100 \mathrm{~W}$ RF power, and mean film thickness varying between $6 \mathrm{~nm}$ and $10 \mathrm{~nm}$. By using this procedure, we obtained metallic nanoparticles dispersed over the nanotube surfaces (Figure 1(b)). The as-obtained sensors were fixed in the microreactors [13] and the electrical connections were made between the contact pads and the reactor pins using the wirebonding method, as schematically shown in Figure 1(c).

2.2. Description of the Microreactors. We fabricated reactors having a small working volume $(100 \mu \mathrm{L})$ in order to evaluate the real characteristic times (response/recovery) of the gas sensors described in Figure 1.

It should be pointed out that the chamber filling times are always incorporated in the total sensor response time, so that, for large chamber volumes $\left(\sim 10^{4} \mathrm{~mL}\right)$, the sensor response times are actually equal to the chamber filling/evacuating times which exceed $10 \mathrm{~s}$ [5]. We compared how the concentration of the input gas changes with time for different chamber volumes at a fixed flow rate of $20 \mathrm{sccm}$ and, for our reactor, the time for attaining $95 \%$ of the desired atmosphere is $\sim 0.3 \mathrm{~s}$ [13]. Thus for detection of fast $(<1 \mathrm{~s})$ processes, reduced $(0.1 \mathrm{~mL}$ or smaller) chamber volume is essential.

Figures 2(a) and 2(b) present images of an open and sealed reactor, respectively, enclosing a chip with four sensitive heads. The test chamber, machined from a single piece of Kovar, is composed by a working cavity (1), four $0.5 \mathrm{~mm}$ diameter electrical pins (2), an upper lid (3), and $1 / 4^{\prime \prime}$ Swagelok-compatible handles placed at diametrically opposed positions of the testing chamber (Figure 2(c)). Due to its $1 / 4^{\prime \prime}$ tubular terminations, the reactor can be easily connected to any vacuum/testing system (Figure 2(a)). More details about this small testing chamber are described elsewhere [13].

2.3. Device Characterization and Measurements. The devices were morphologically characterized by field emission scanning electron microscopy (FE-SEM, NOVA 200 Nanolab, FEI Co.) and Raman spectroscopy (NTEGRA, NT-MDT) by exciting the spectra with a $473 \mathrm{~nm}$ laser line. Using the reactors described above, the electrical characterization of the sensors was performed in vacuum or pure oxygen atmosphere using $20 \mathrm{sccm}$ mass flow controllers (M100B, MKS Instruments) and a 2636A Keithley source-meter in the $10^{-5}$ to $10^{-2} \mathrm{~W}$ working power interval.

\section{Results and Discussion}

3.1. Morphological Characterization. Figure 3(a) shows FESEM images of the layout of the gas sensors. The chips have four sensors (Inset in Figure 3(a)) and each element 


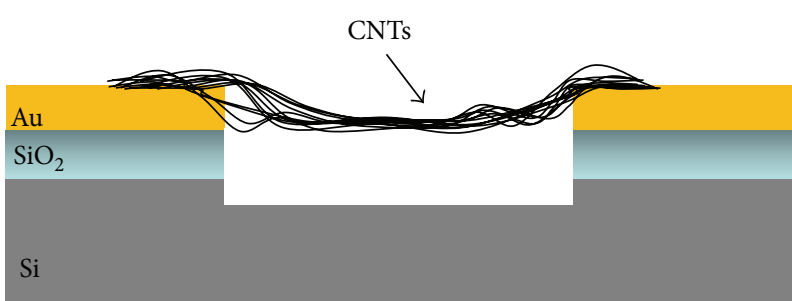

(a)

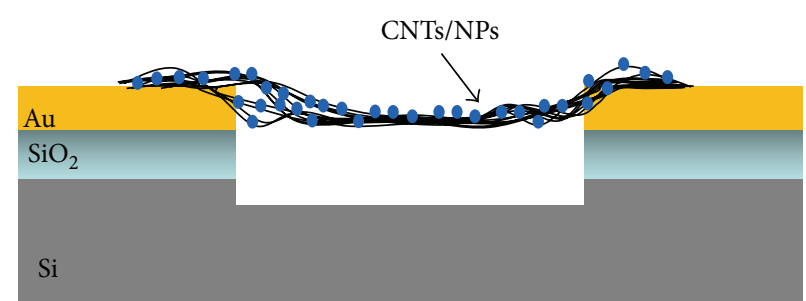

(b)

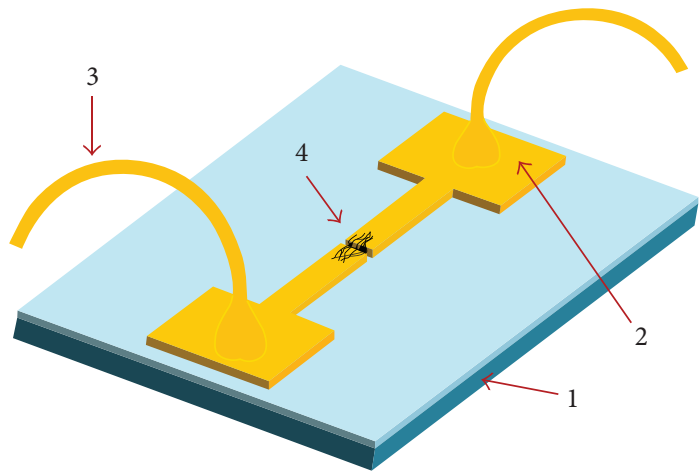

(c)

FIGURE 1: Schematic drawings of the chemical resistor sensor configuration based on suspended nanostructures bridging the gap between the electrodes: before (a) and after (b) metallic nanoparticles deposition by sputtering; (c) oxidized silicon substrates (1), gold patterned electrodes (2), wire-bonding connections (3), and the gap with the carbon nanotubes (4) compose the devices tested using the microreactors.

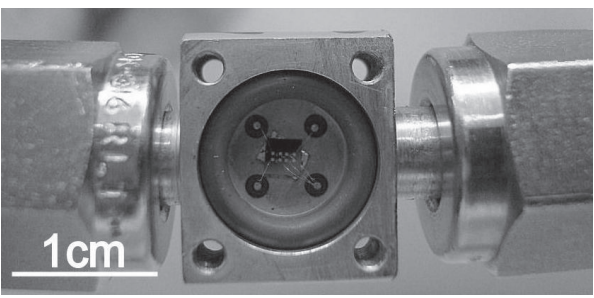

(a)

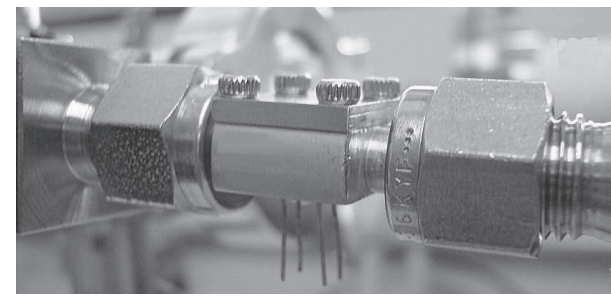

(b)

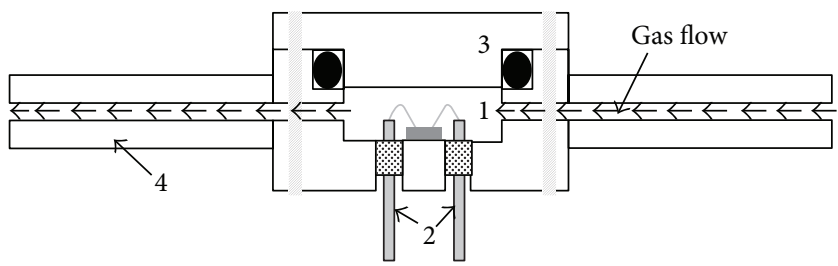

(c)

Figure 2: Image of an open (a) and sealed (b) reactor, enclosing a chip with four sensors, connected to the vacuum system. (c) Schematic drawing of the reactor having a volume of $100 \mu \mathrm{L}$. The working cavity (1), the electric contact pins (2), the upper lid (3), and the connecting tubes (4) are all molded in Kovar.

is composed of two $200 \times 200 \mu \mathrm{m}$ gold pads linked by a $300 \mu \mathrm{m}$ long and $20 \mu \mathrm{m}$ wide gold line. In the central region of each line connecting the pads, a $5 \mu \mathrm{m}$ deep and $1 \mu \mathrm{m}$ wide gap is milled by FIB (Figure 3(a)). Depending on the DEP deposition conditions and on the concentration of the suspension, sensors composed by one (Figure 3(b)) or several (Figure 3(c)) nanotubes can be obtained. For the device presented in Figure 3, the deposition conditions were a frequency of $100 \mathrm{kHz}$, a $3 \mathrm{~V}$ peak-to-peak voltage, and a deposition time of 75 seconds (Figure 3(b)) and 3 minutes (Figure 3(c)).

The gap between the electrodes allowed us to fabricate sensors in a chemical resistor configuration based on suspended carbon nanotubes decorated with metallic nanoparticles. Since we use the Joule effect to heat locally our devices, the self-heating of the suspended nanotubes is much stronger when compared with the supported configuration, where the nanostructures are in direct thermal 


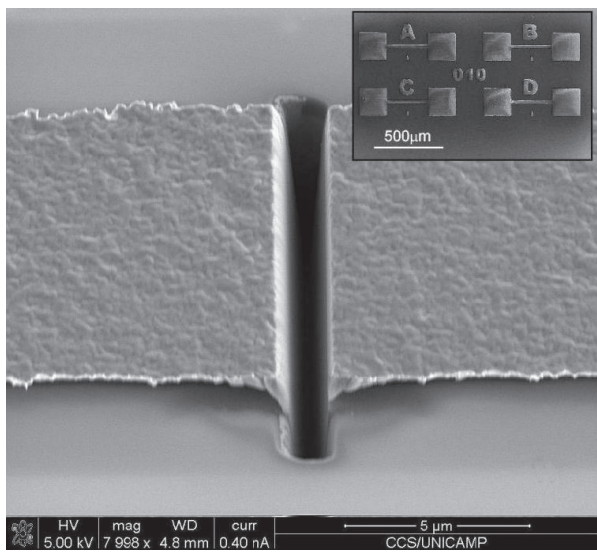

(a)

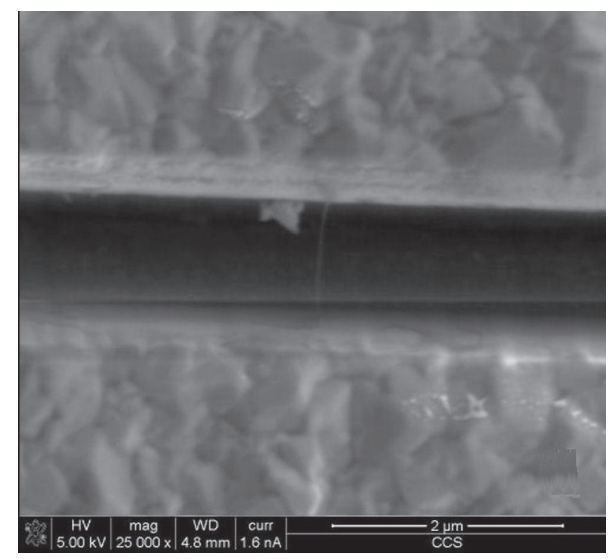

(b)

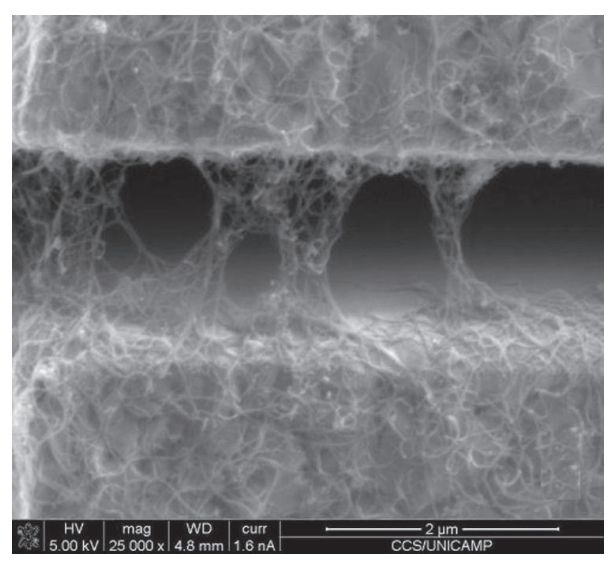

(c)

FIgURE 3: FE-SEM images of (a) a gap of $5 \mu \mathrm{m}$ deep and $1 \mu \mathrm{m}$ wide milled by FIB in the central region of the line connecting the pads. The inset shows a chip composed of four gold electrodes; a sensor is composed of one (b) or several (c) nanotubes depending on the deposition conditions.

contact with the oxidized silicon substrate and the heat can dissipate through it. This self-heating was shown to increase the temperature of suspended nanotubes up to $400-500^{\circ} \mathrm{C}$, thereby strongly enhancing the sensors response to gases when nanoparticle-decorated nanotubes are used $[5,6]$.

Figure 4(a) shows the Raman image of the $G$ band mode (at $1580 \mathrm{~cm}^{-1}$ shown in inset to Figure 4(a)) for the pristine multiwall carbon nanotubes deposited over the gap separating the pads. We can observe a strong D band signal at about $1359 \mathrm{~cm}^{-1}$, which is attributed to defects, thus showing that the nanotubes have structural defects [14].

After the DEP deposition, the devices were introduced in the sputtering system and $\mathrm{Ti}$ or $\mathrm{Cu}$ nanoparticles were deposited over the nanotubes. Figure 4(b) shows FE-SEM images of $\mathrm{Cu}$ decorated CNTs using an RF power of $100 \mathrm{~W}$, an argon pressure of $2 \times 10^{-2}$ mbar, and a film thickness of $10 \mathrm{~nm}$. One can see that the surface of the nanotube is covered with nanoparticles having a narrow size distribution with a mean diameter of $\sim 10 \mathrm{~nm}$. The energy dispersive spectroscopy (EDS) measurements (Figure 4(c)), carried out on the central part of the decorated nanotube (see Figure 4(b)), indicate that the weight percentage of $\mathrm{Cu}$ is near $20 \%$, confirming the decoration of the nanotubes by metallic particles.
3.2. Gas Sensing Response. In order to test the sensors embedded in the microreactors, a small vacuum system composed of a rotary pump, a turbo molecular pump, two pressure sensors, mass flow controllers, and a gas distribution chamber was mounted. Details about microreactor design and vacuum system configuration can be found elsewhere [13].

It is important to mention here that much research was conducted onto titanium oxide gas sensibility, especially in thin film [15] and nanostructured form [16], but few reports register the sensing behavior of metal nanoparticles. Here we study carbon nanotubes decorated with metallic nanoparticles (titanium and copper) where the heating is localized, being achieved by Joule effect. We thus have the self-heated nanotubes as heater and transducer for the decorating nanoparticles.

Gas sensing measurements were carried out in the pressure range from $1 \times 10^{-4}$ to $1 \times 10^{3}$ mbar. The smallest pressure was achieved by using the turbo pump attached to the vacuum system and the highest one by closing all the valves and isolating the reactor, filled with $\mathrm{O}_{2}$, from the system. Figures 5,6 , and 7 show some of the results obtained. The difference in the sensors initial resistance values is due to differences in the number of nanotubes connecting the pads. 


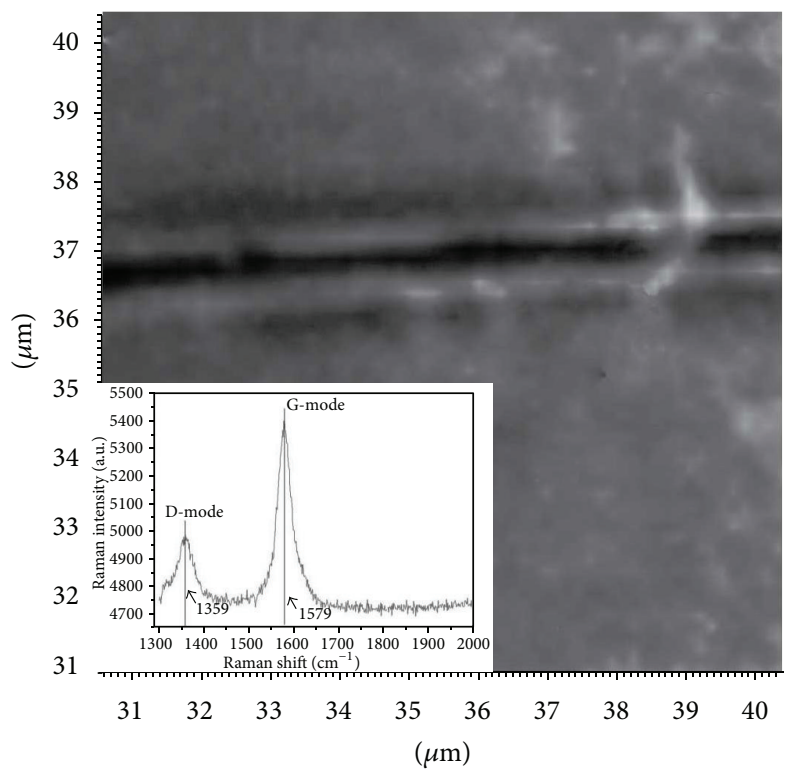

(a)

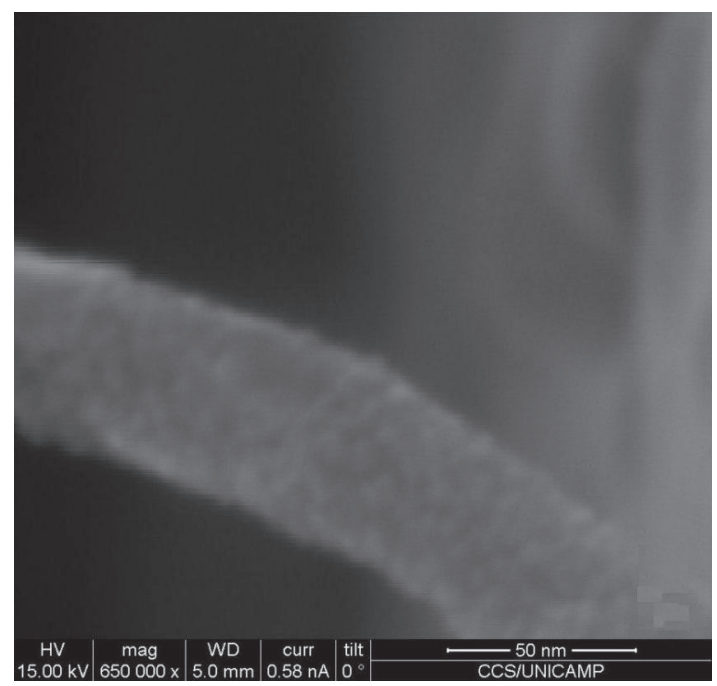

(b)

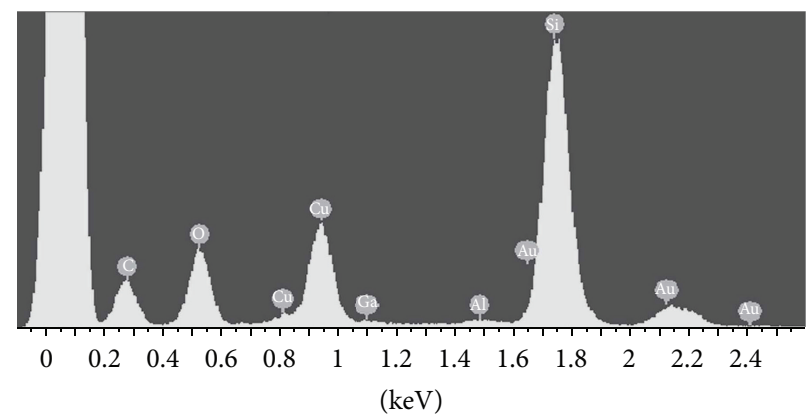

(c)

FIgURE 4: (a) Raman image of a pristine carbon nanotube deposited by DEP over gold electrodes, excited with a $473 \mathrm{~nm}$ laser line. The inset shows Raman spectrum for carbon nanotube D and G lines; (b) FE-SEM images of Cu decorated CNTs and (c) results of EDS measurements carried out on the pink rectangular area marked in the FE-SEM image in (b).

Depending on the DEP conditions used for deposition, we obtained samples composed of few (as pointed further in the text, just two) or a larger number of nanotubes (more than 20) aligned over the gap. It should also be pointed out that, in our previous report [13], we also conducted studies of gas sensors based on pristine nanotubes. This research highlighted the low sensibility and the lack of selectivity to the target gas, these devices being more suitable for pressure sensing applications.

Figure 5(a) shows the response to oxygen pulses for the device based on Ti-decorated carbon nanotubes using a working power of $3 \times 10^{-5} \mathrm{~W}$ and testing pressures of $1.5 \mathrm{mbar}$ and $1 \times 10^{3} \mathrm{mbar}$. As one can see, for $1.5 \mathrm{mbar}$ pressure, the device response is quite low and the recovery is slow. When the oxygen pressure is increased in the reactor (the valve that isolates the reactor from the vacuum system is closed) the response rises significantly. For better illustrating this response, one gas cycle was applied to the sensor (Figure 5(b)) using the same working power. The sensitivity is quite high, with the device reaching stability (around $100 \mathrm{~s}$ testing time).
Nevertheless, the recovery is slow in the time interval used for the tests.

Even though a thermal treatment was applied to all the sensors, during measurements we frequently noticed improving of the electrical contacts between nanotubes and metal electrodes (drop in resistance). This can be observed, for instance, in Figure 5(b) for $110 \mathrm{~s}$. We are currently studying several routes for achieving an improved contact between the nanotubes and the leads.

By comparing the two types of metallic ( $\mathrm{Ti}$ and $\mathrm{Cu}$ ) nanoparticles used (Figure 6), it can be noticed that their behavior is quite similar, as expected. It is important to point out that both devices are composed of only two nanotubes bridging the gold electrodes (FE-SEM images not shown here). For the sensor decorated with Ti (Figure 6(a)), the sensitivity towards $\mathrm{O}_{2}$ is good, with a response time of $\sim 3 \mathrm{~s}$. A stronger response is exhibited by the $\mathrm{Cu}$ decorated device (Figure 6(b)), but with a slower response time of $\sim 6 \mathrm{~s}$. The response time of the samples was calculated as the time necessary to reach $90 \%$ of the final value. For both devices the 


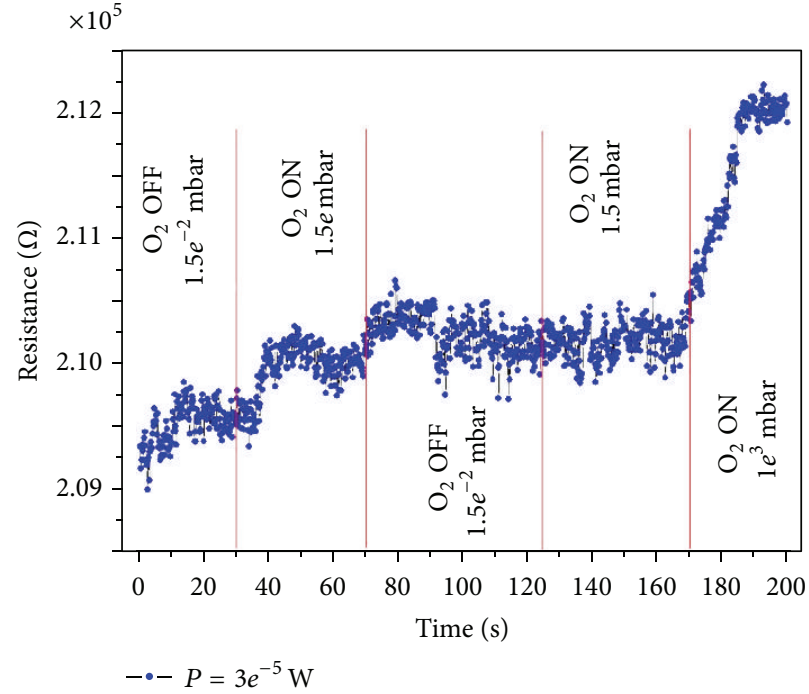

(a)

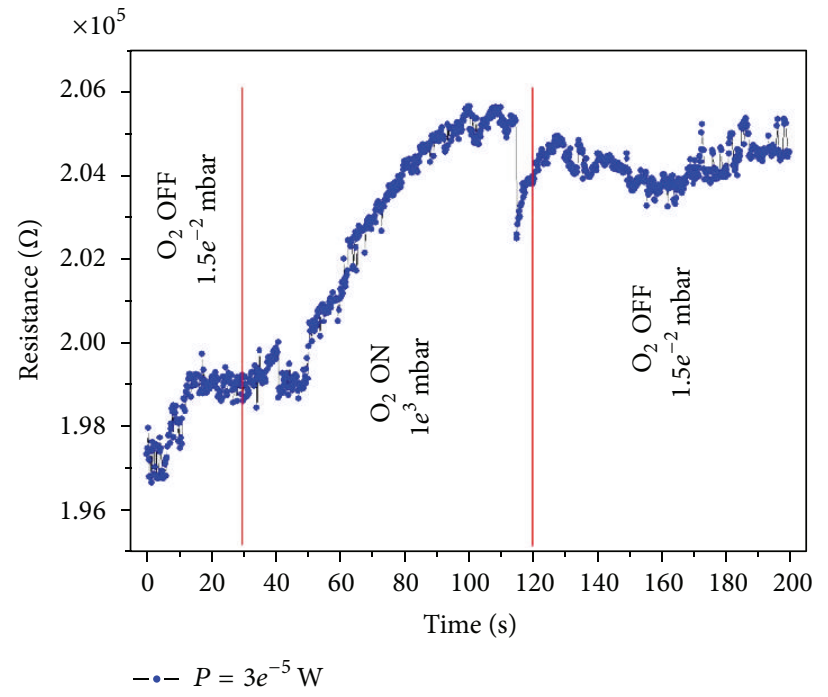

(b)

FIGURE 5: Response towards oxygen for a Ti nanoparticle-decorated carbon nanotube-based sensor submitted to (a) several gas cycles and (b) one gas cycle using different testing pressures.

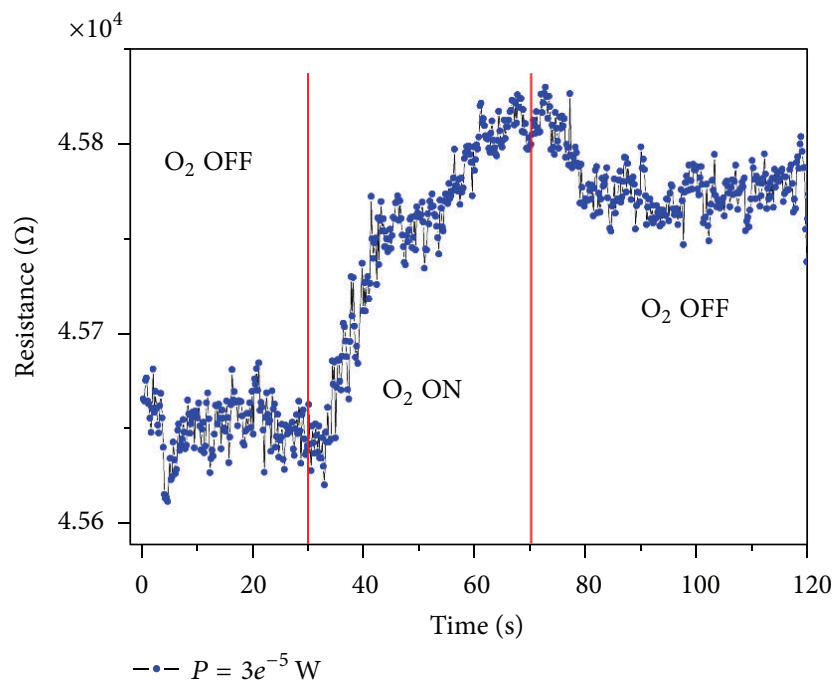

(a)

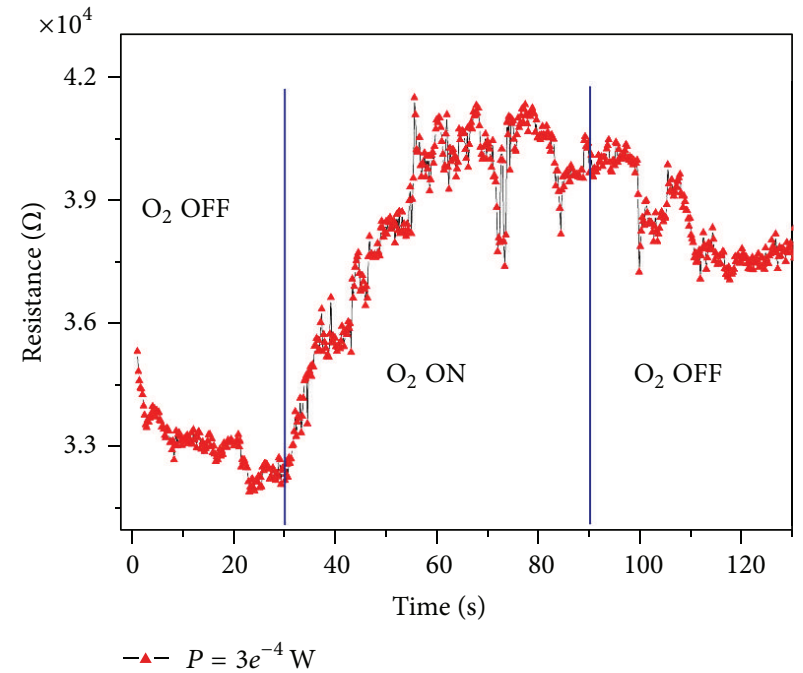

(b)

FIgURE 6: Resistance change with time for $\mathrm{Ti}(\mathrm{a})$ and $\mathrm{Cu}(\mathrm{b})$ nanoparticle decorated sensors. The gas flow used for testing was $20 \mathrm{sccm}$.

recovery is slow, with the sensors not reaching the resistance initial value in the time interval used for measurements. The slow response time as well as the low recovery is probably due to the low working temperature the sensors operate on.

In order to verify this hypothesis, we increased the working power by two (Figure 7(a)) and three (Figures 7(b) and $7(\mathrm{c})$ ) orders of magnitude for the devices composed of CNTs/Ti nanoparticles. It is evident that the response increased and the sensor recovery was enhanced. Due to a higher working temperature, the oxygen probably desorbs faster from the nanostructure surfaces, with the devices reaching their initial resistance values. Even though the sensor was cycled with longer $\mathrm{O}_{2}$ exposition times, it recovers for both cycles. For higher working power (Figure 7(b)), the resistance does not return to its initial value, most likely due to nanoparticle bulk oxidation during sensor testing.

The sensor response seems to be composed of two distinct phases: a rapid increase in the resistance $(<1 \mathrm{~s})$ due to oxygen adsorption followed by nanoparticle surface oxidation and a slow process $(\sim 5 \mathrm{~s})$ most probably related to the oxygen diffusion within the nanoparticles followed by bulk Ti oxidation, as can be seen in Figure 7(c).

\section{Conclusions}

In summary, we fabricated and tested sensors based on a few multiwall carbon nanotubes decorated with metallic ( $\mathrm{Cu}$ and Ti) nanoparticles with high sensitivity and reproducibility 


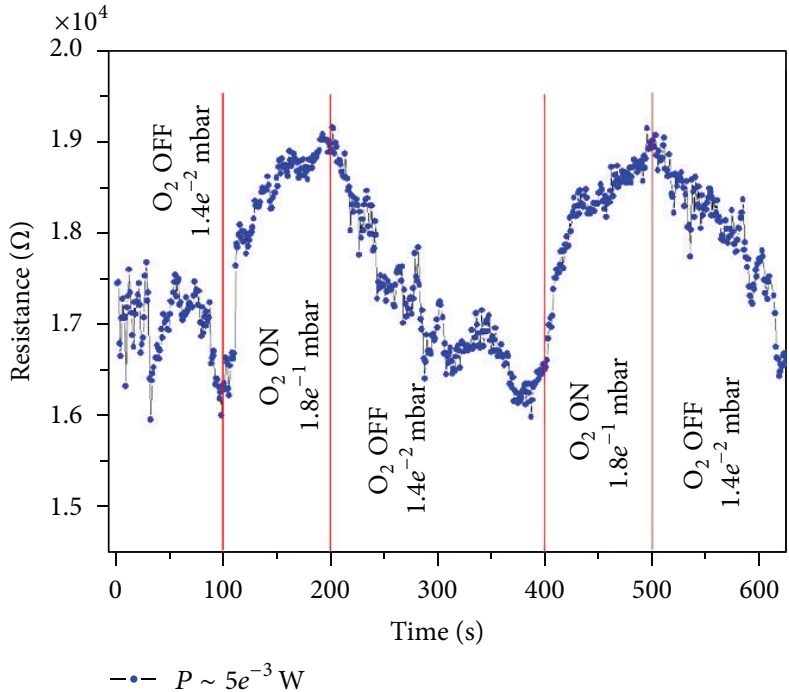

(a)

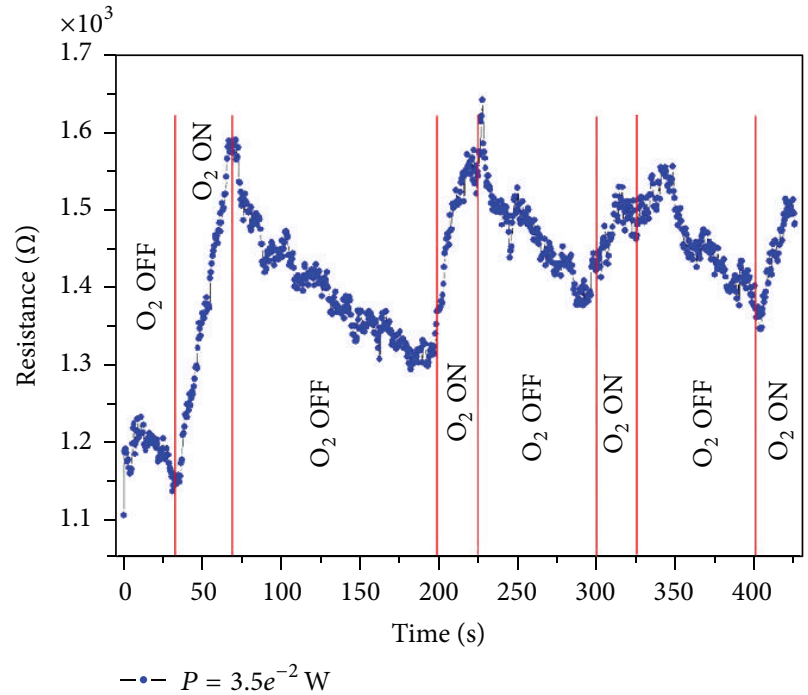

(b)

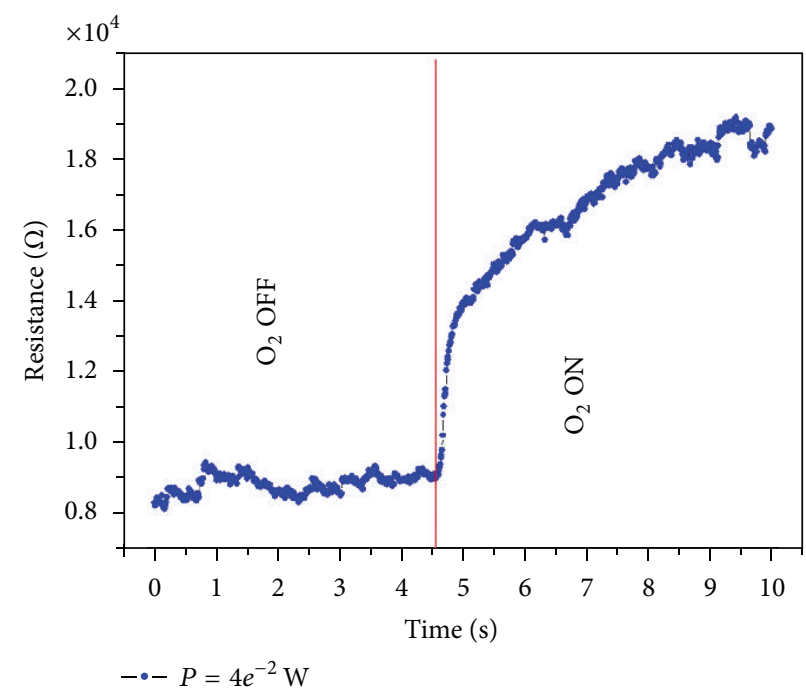

(c)

FiguRE 7: Sensitivity towards $10 \mathrm{sccm}$ oxygen flow for Ti nanoparticle decorated carbon nanotube-based sensors for working powers of $5 \times$ $10^{-3} \mathrm{~W}(\mathrm{a}), 3.5 \times 10^{-2} \mathrm{~W}(\mathrm{~b})$ showing sensor reproducibility to oxygen cycling, and $4 \times 10^{-2} \mathrm{~W}$ (c) showing the two distinct response processes. The testing pressure range for (b) and (c) is the same as the one used in (a).

towards oxygen. The FE-SEM and Raman spectroscopy results showed that we successfully deposited carbon nanotubes, by DEP, over gold patterned electrodes. The sputtering decoration of these nanostructures with metallic nanoparticles, as confirmed by EDS and FE-SEM, proved to be a suitable strategy for enhancing their sensitivity towards gases.

As the chamber filling times are always included in the total sensor response, we fabricated reactors having a small working volume $(100 \mu \mathrm{L})$ in order to see the real characteristic operation times (response/recovery) of the devices. The design and the approach adopted for fabricating the devices proved to be adequate for obtaining the desired small-volume atmosphere-controlled reaction chamber. Due to reduced reactor volume, it was possible to measure response times towards oxygen detection shorter than $1 \mathrm{~s}$ for Ti nanoparticledecorated CNT-based sensors. Moreover, it was possible to see the two distinct processes that occur during gas cycling: a rapid surface oxidation process due to oxygen adsorption and reaction with nanoparticle surfaces and a slow process $(\sim 5 \mathrm{~s})$ most probably related to the oxygen diffusion within the particles followed by bulk Ti oxidation. Work is now in progress for a better understanding of the oxidation processes that occur during sensor cycling as well as for nanocontact improvement.

\section{Conflict of Interests}

The authors declare that there is no conflict of interests regarding the publication of this paper. 


\section{Acknowledgments}

J. V. Silveira and R. Savu would like to acknowledge the financial support of CAPES (Grant no. 068/2007 PROCAD program and Rede Nanobiotec) and FAPESP (Grant no. 2009/14600-4) Brazilian foundations, respectively. All the authors acknowledge the financial support of FAPESP and CNPq Brazilian foundations. A. G. Souza Filho acknowledges support from CNPq (Grant 307317/2010-2), INCT NanoBioSimes, and FUNCAP (PRONEX PR2-005400022.01.00/11).

\section{References}

[1] K. Tanaka, T. Yamabe, and K. Fukui, The Science and Technology of Carbon Nanotubes, Elsevier Science, Oxford, UK, 1999.

[2] S. A. Wilson, R. P. J. Jourdain, Q. Zhang et al., "New materials for micro-scale sensors and actuators: an engineering review," Materials Science and Engineering: R: Reports, vol. 56, no. 1-6, pp. 1-129, 2007.

[3] A. Loiseau, P. Launois, P. Petit, S. Roche, and J.-P. Salvetat, Understanding Carbon Nanotubes, vol. 677 of Lecture Notes in Physics, Springer, Berlin, Germany, 2006.

[4] Q. Cao and J. A. Rogers, "Ultrathin films of single-walled carbon nanotubes for electronics and sensors: a review of fundamental and applied aspects," Advanced Materials, vol. 21, no. 1, pp. 2953, 2009.

[5] R. V. Gelamo, F. P. Rouxinol, C. Verissimo, A. R. Vaz, M. A. B. de Moraes, and S. A. Moshkalev, "Low-temperature gas and pressure sensor based on multi-wall carbon nanotubes decorated with Ti nanoparticles," Chemical Physics Letters, vol. 482, no. 4-6, pp. 302-306, 2009.

[6] R. V. Gelamo, F. P. Rouxinol, C. Verissimo, M. A. De Bica Moraes, and S. A. Moshkalev, "Gas and pressure sensors based on multi-wall carbon nanotubes: study of sensing mechanisms," Sensor Letters, vol. 8, no. 3, pp. 488-492, 2010.

[7] P. Abgrall and N. T. Nguyen, "Nanofluidic devices and their applications," Analytical Chemistry, vol. 80, no. 7, pp. 2326-2341, 2008.

[8] S. Mubeen, J.-H. Lim, A. Srirangarajan, A. Mulchandani, M. A. Deshusses, and N. V. Myung, "Gas sensing mechanism of gold nanoparticles decorated single-walled carbon nanotubes," Electroanalysis, vol. 23, no. 11, pp. 2687-2692, 2011.

[9] K. Li, W. Wang, and D. Cao, "Metal (Pd, Pt)-decorated carbon nanotubes for CO and NO sensing," Sensors and Actuators B: Chemical, vol. 159, no. 1, pp. 171-177, 2011.

[10] A. A. Koós, R. J. Nicholls, F. Dillon et al., “Tailoring gas sensing properties of multi-walled carbon nanotubes by in situ modification with Si, P, and N," Carbon, vol. 50, no. 8, pp. 28162823, 2012.

[11] W. C. Shin and R. S. Besser, "A micromachined thin-film gas flow sensor for microchemical reactors," Journal of Micromechanics and Microengineering, vol. 16, no. 4, pp. 731-741, 2006.

[12] L. Dai, P. Soundarrajan, and T. Kim, "Sensors and sensor arrays based on conjugated polymers and carbon nanotubes," Pure and Applied Chemistry, vol. 74, no. 9, pp. 1753-1772, 2002.

[13] R. Savu, J. V. Silveira, A. Flacker et al., "Micro-reactors for characterization of nanostructure-based sensors," Review of Scientific Instruments, vol. 83, no. 5, Article ID 055104, 2012.

[14] M. S. Dresselhaus, A. Jorio, A. G. S. Filho, and R. Saito, "Defect characterization in graphene and carbon nanotubes using Raman spectroscopy," Philosophical Transactions of the Royal Society A, vol. 368, no. 1932, pp. 5355-5377, 2010.

[15] M. A. Ponce, R. Parra, R. Savu et al., "Impedance spectroscopy analysis of $\mathrm{TiO}_{2}$ thin film gas sensors obtained from waterbased anatase colloids," Sensors and Actuators, B: Chemical, vol. 139, no. 2, pp. 447-452, 2009.

[16] V. Galstyan, E. Comini, G. Faglia, and G. Sberveglieri, “ $\mathrm{TiO}_{2}$ nanotubes: recent advances in synthesis and gas sensing properties," Sensors (Switzerland), vol. 13, no. 11, pp. 14813-14838, 2013. 

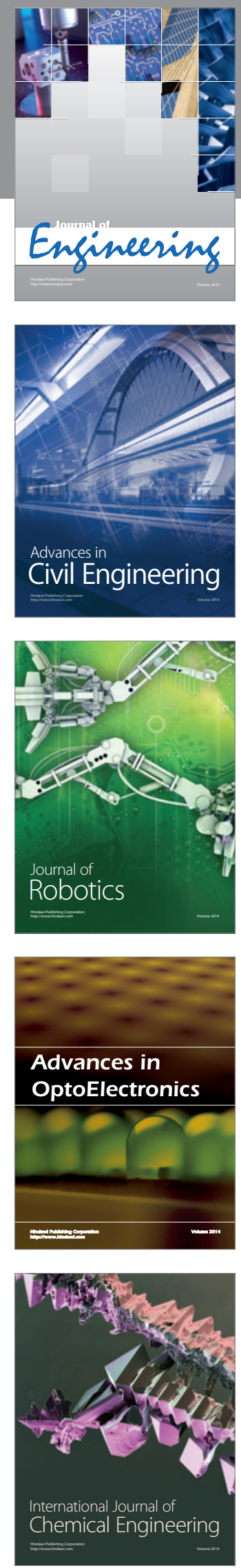

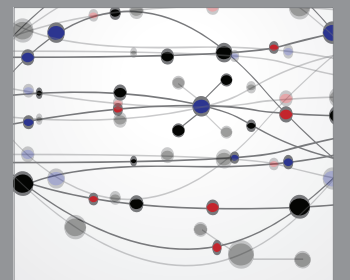

The Scientific World Journal
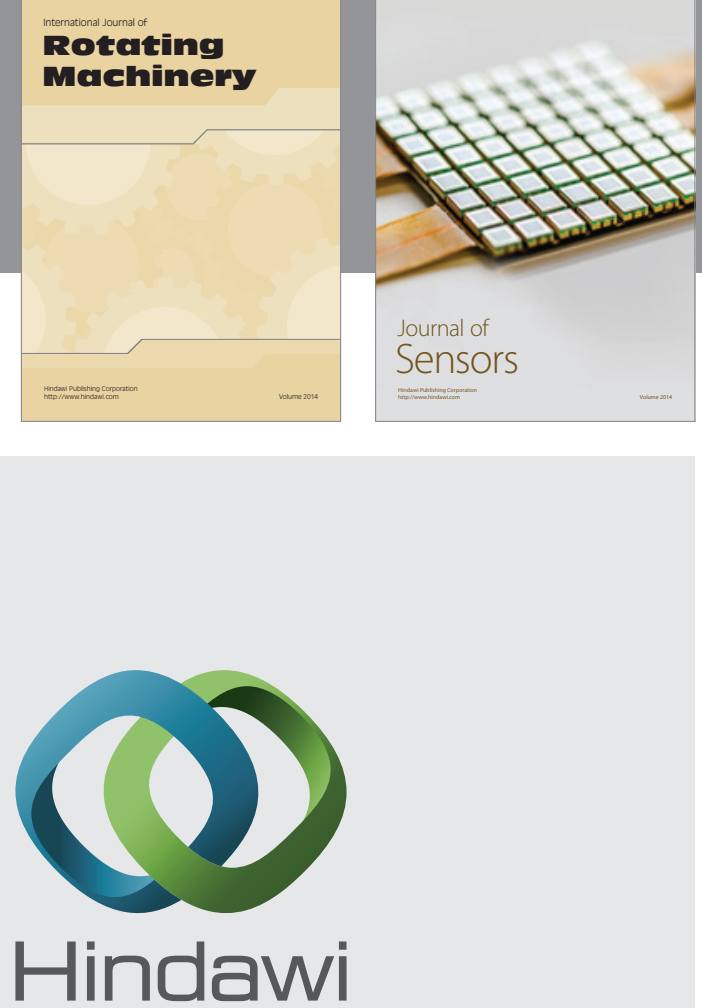

Submit your manuscripts at http://www.hindawi.com
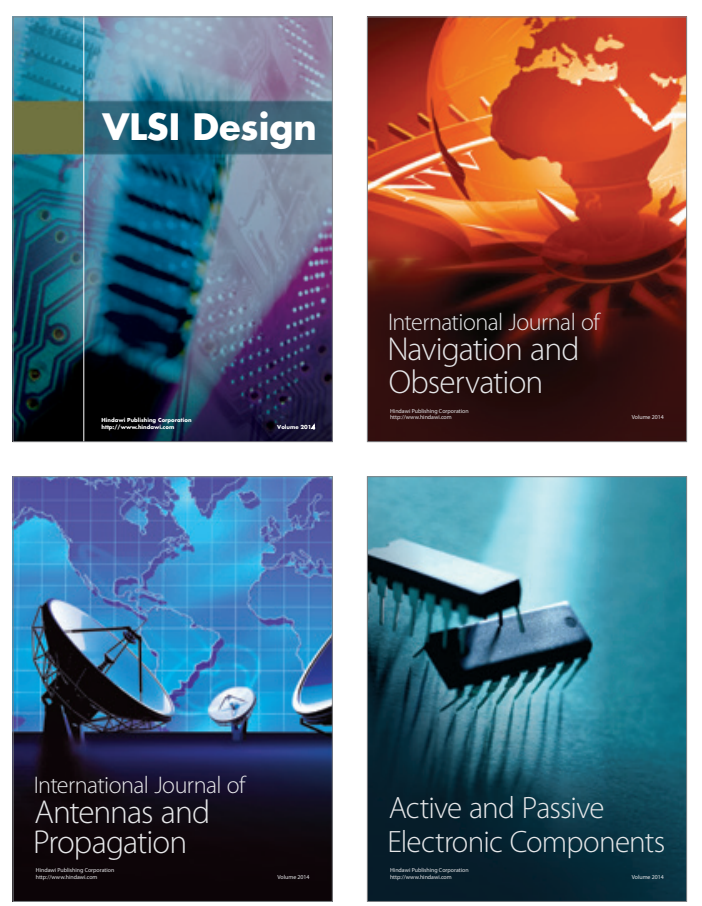
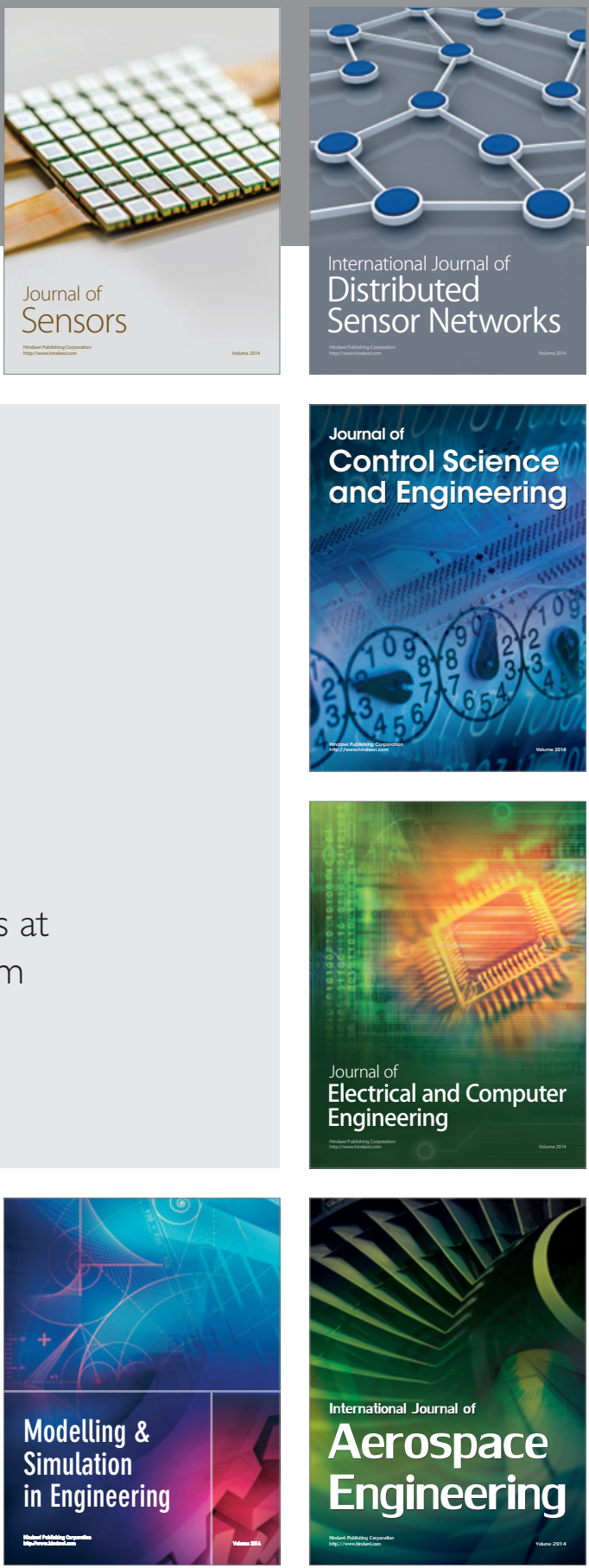

Journal of

Control Science

and Engineering
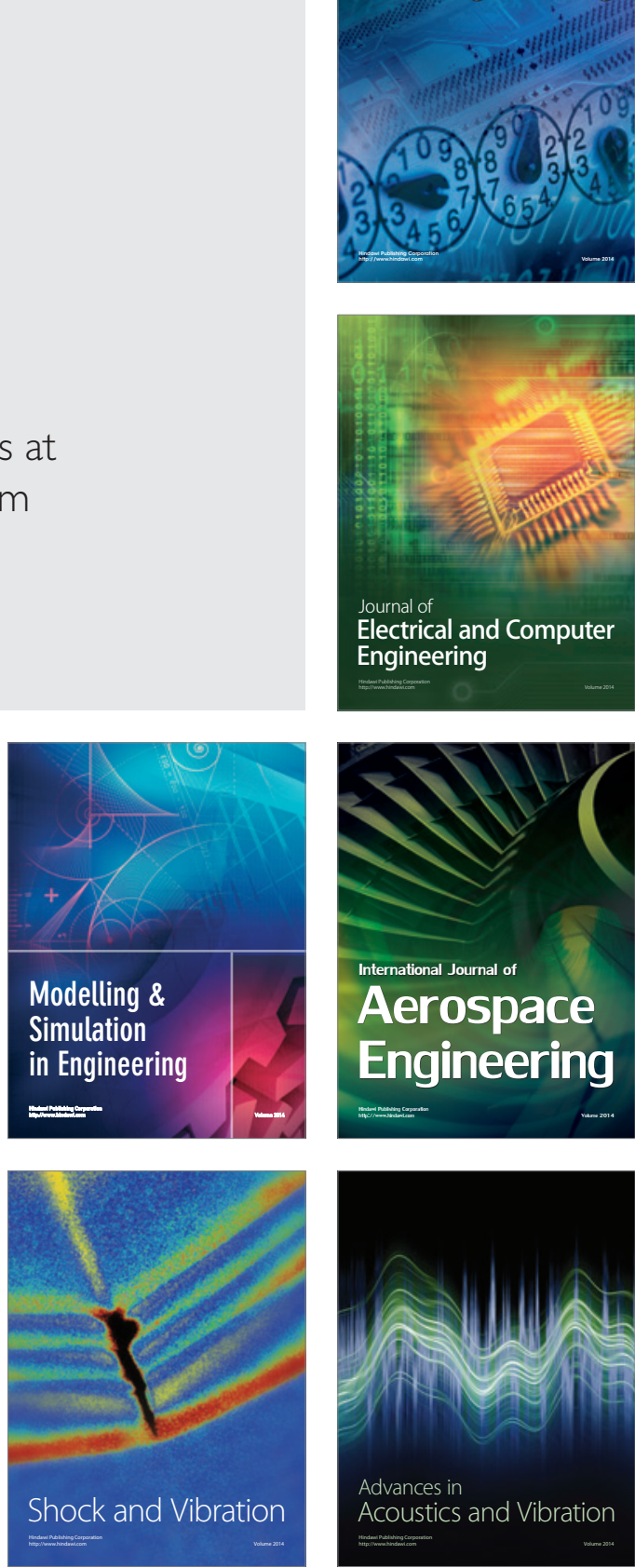